\title{
Coupling, Q-factor, and Integration Aspects of Microsphere Applications
}

\author{
V.S.Ilchenko, X.S.Yao, L.Maleki \\ 298-100 Jet Propulsion Laboratory, California Institute of Technology \\ 4800 Oak Grove Dr. Pasadena CA 91109
}

With suggested applications varying from microlaser and cavity QED through optical locking of diode lasers to modulators and sensors [1-3], high-Q silica microspheres with whispering-gallery (WG) modes so far remain the subject of tabletop feasibility demonstrations. Despite the uniquely high quality-factor and submillimeter dimensions suitable for tight packaging, this novel type of high-finesse cavity still has to be adapted to fiber- and integrated-optic hardware.

In the visible and near infrared-band experiments $(633-850 \mathrm{~nm})$ measuring the ringdown time $\tau$ of free oscillations, $Q=(0.6$ to 0.8$) \times 10^{10}$ has been obtained in silica spheres of diameter $\sim 800 \mu \mathrm{m}$ [4] (corresponding $\tau=3$ to $4 \mu \mathrm{s}$ ). It was proved that under normal laboratory conditions, quality-factor is subject to deterioration within several-minute scale down to $(2 \ldots 3) \times 10^{9}$. The responsible mechanism was identified as adsorption of a monolayer of atmospheric water, so that preservation of the ultimate $Q$ requires manipulation in dry environment, or fast packaging into sealed devices. Larger $Q$ can be expected closer to minimum of attenuation in fused silica $\alpha$ $=0.2 \mathrm{~dB} / \mathrm{km} ; Q \quad \geq 1 \times 10^{11}$ at $\lambda=1.55 \mu \mathrm{m}$, with corresponding energy storage time $\tau \sim 0.1 \mathrm{~ms}$. Experiments are currently underway to determine whether this high $Q$ can be realized experimentally. The evident difficulty is that $\mathrm{OH}$-related optical absorption has its peaks located near the reported minimum of attenuation in silica. We can also mention here that some of proposed fiber materials, yet not ready for fiber drawing, have been predicted to have smaller attenuation than fused silica and may be suitable for microsphere fabrication (sodium-magnesium silicate glass, $\alpha=0.06 \mathrm{~dB} / \mathrm{km}[5]$ ).

WG modes possess very small radiative loss (it does not prevent $Q \sim 10^{20}$ and more) and therefore are electromagnetically isolated and cannot be excited by free-space beams. If no modification (such as grating) is made on the sphere surface, the coupling has to be provided by an appropriate near-field device. A systematic theoretical approach has been recently developed to quantify the performance of near-field couplers for WG modes [6]. Efficient coupling can be obtained upon fulfillment of two main conditions: 1)mode matching and 2)sufficient coupling strength to provide the buildup of a WG mode with a given intrinsic loss. In other words, the wave in the coupler has to be phase-matched and sufficiently overlapped with the WG mode to provide enough buildup in the cavity. If the coupling efficiency is characterized by the fractional depth $K^{2}$ of the resonance dip in intensity transmittance observed upon varying the frequency of the exciting wave in the coupler,
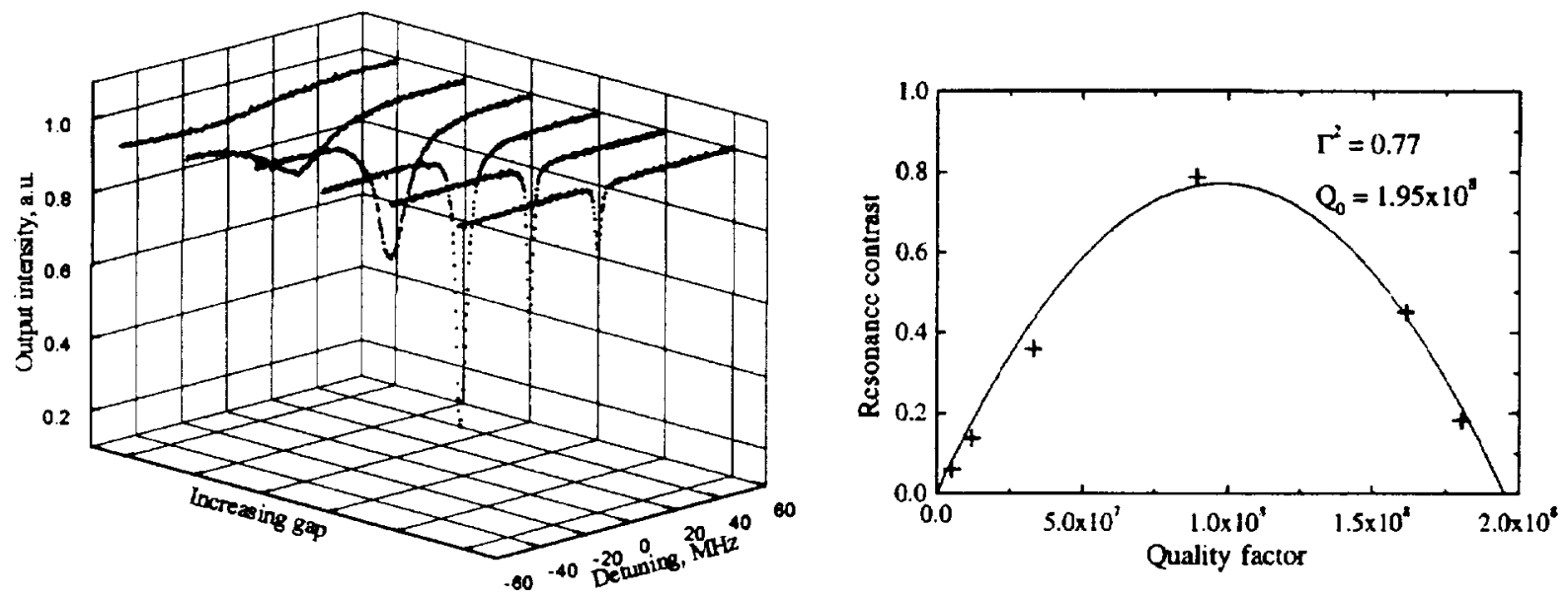

Fig.1. Effect of variable coupling (increasing microsphere-coupler gap) on the loaded quality-factor and contrast of WG mode resonance observed as depletion peak in transmitted power. Single prism coupler; wavelength $633 \mathrm{~nm}$; fused-silica microsphere with diameter $270 \mu \mathrm{m}$; unloaded $Q_{0} \approx 2 \times 10^{8}$ 
then it can be expressed in the following way through $Q_{c}$ and the intrinsic quality-factor of the mode $Q_{o}$ :

$$
K^{2}=4 Q_{0} Q_{r} \Gamma^{2} /\left(Q_{n}+Q_{r}\right)^{2}
$$

With complete mode matching $\Gamma=1$, the critical coupling $K^{2}=1$, or $100 \%$ absorption of the input wave in resonance, is possible when $Q_{c}$ can be made equal or smaller than $Q_{o}$. Both contrast of the visible resonance $K$ and the loaded quality-factor $Q_{L}=\left(Q_{o}^{-1}+Q_{c}^{-1}\right)^{-1}$ can be adjusted by varying the gap between the coupler device and the sphere, giving a unique (in optics) opportunity to easily control the bandwidth of the cavity. Typical evolution of the observed absorption resonance with increasing of the gap is illustrated in Fig.l. Results are obtained with prism coupler at the wavelength $633 \mathrm{~nm}$. The "disappearance" of the resonance dip in overloaded regime with wellmatched coupler does not mean reduced energy exchange; it reflects only the well known fact in RF engineering that maximum absorption at resonance requires that coupling loss be equal to intrinsic cavity loss critical $Q_{c}=Q_{o}$, or $Q_{l}$ $=1 / 2 Q_{o}$. If $Q_{c} \ll Q_{o}$, the power is recycled in the mode and is fed back to the coupler with shifted overall phase.

At present moment, in addition to the well-known prism coupler with frustrated total internal reflection [13,6], few attempts have been reported to directly couple a sphere to an optical fiber. However these fiber couplers had either limited efficiency due to residual phase mismatch (side-polished bent fiber coupler [7]), or still appreciable size including fragile core-to-cladding transformers (tapered fiber coupler [8]). Recently, we have demonstrated a new and simple method of direct fiber coupling to high-Q WG modes, which in essense is a hybrid of waveguide and prism coupler. A close-up of the experimental setup with microsphere and two couplers is shown in Fig.2a. The tip of a single-mode fiber is angle-polished under steep angle. Upon incidence on the angled surface, the light propagating inside the fiber core undergoes total internal reflection and escapes the fiber. With the sphere positioned in the range of the evanescent field from the core area, the configuration provides efficient energy exchange in resonance between the waveguide mode of single-mode fiber and the whispering-gallery mode in the sphere. The angle of the polish is chosen to secure the phase matching requirement: $\Phi=\arcsin \left(n_{\text {sphere }} / n_{\text {fiber }}\right)$. Here $n_{\text {fiber }}$ stands for the effective refraction index to describe the guided wave in the fiber core truncation area, and $n_{\text {sphere }}$ stands for the effective refraction index to describe azimuthal propagation of WG modes (considered as closed waves undergoing total internal reflection in the sphere), see plot in Fig. 3. Since the linear dimensions of the angle-
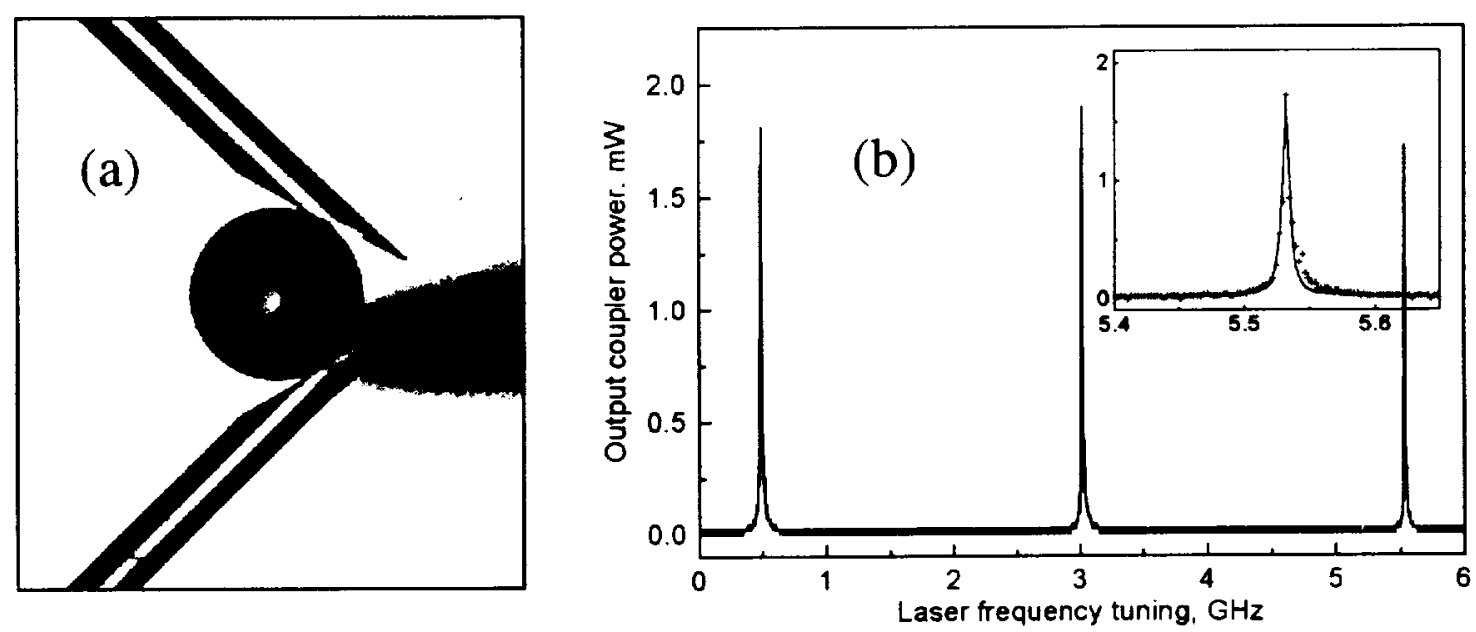

Fig.2. Close-up view (a) and optical transmission characteristic (b) of a microsphere with two side-polished fiber couplers. Input power $7.5 \ldots 8.3 \mathrm{~mW}$; maximum transmission at resonance $\sim 23.5 \%$ (fiber-to-fiber loss $6.3 \mathrm{~dB}$ ); $Q_{L}>3 \times 10^{7}$ at $1550 \mathrm{~nm}$; sphere diameter $405 \mu \mathrm{m}$. Unloaded $Q_{o} \approx 1.2 \times 10^{8}$. Fiber: SMF-28, polished at $\sim 76^{\circ}$.

cut core area match well the area of evanescent field overlap, the new system is equivalent to a prism coupler with eliminated collimation/focusing optics. The effective refraction index to describe the azimuthal propagation of WG modes near the surface of the sphere can be calculated, for example, on the basis of asympthotic expressions [9] for WG mode frequencies $\omega_{q}$, where $n_{\text {sphere }}=2 c l / D \omega_{q}$. Single coupler efficiency in our experiment was more than $60 \%$, comparable with the best reported results for prism coupler $(78 \%)$ and fiber taper $(90 \%)$; total fiber-to-fiber transmission at resonance $23 \%$ (insertion loss $6.3 \mathrm{~dB}$ ). The demonstrated simple "pigtailing" of the microspheres 
will lead to their wider use in fiber optics, enabling the realization of a whole class of new devices ranging from ultra-compact narrow band filters and spectrum analyzers and high-sensitivity modulators and sensors, to compact laser frequency stabilization schemes and opto-electronic microwave oscillators.

Apart from "discrete element" fiber optics applications, microspheres present further challenge for true integration with planar optics devices. Very high $\mathrm{Q} \geq$ $10^{8} \ldots 10^{9}$, uncommon for existing planar microcavities, would open way for sub$\mathrm{kHz}$-linewidth integrated laser sources [3] and single-chip microwave optoelectronic oscillators [10]. The ultimate goal is the replacement of laboratory hand preparation of spheres (fusion of silica preforms in oxhydric flame or $\mathrm{CO}_{2}$ laser beam) by

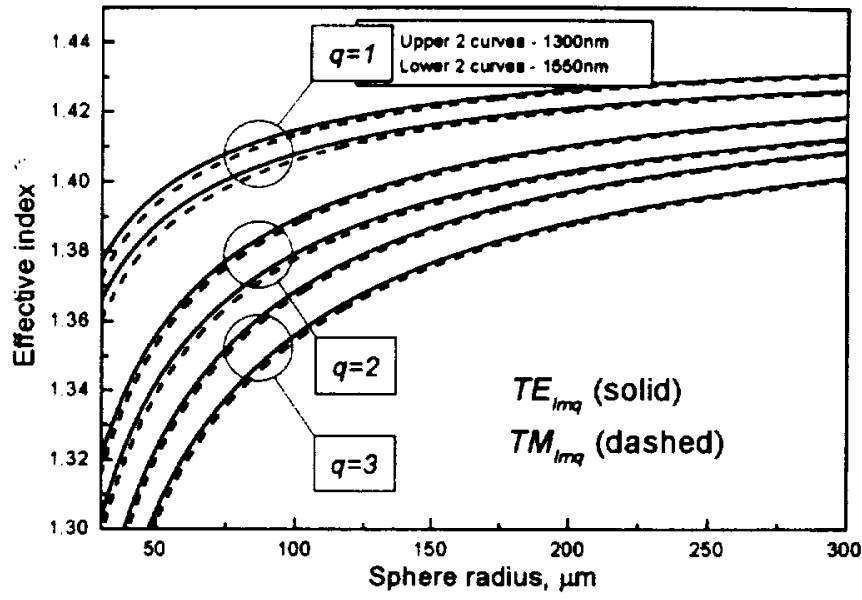

Fig.3. Effective index for WG mode azimuthal propagation (based on mode frequency approximation by C.C.Lam et al [9]) appropriate microfabrication technology. It is worth to note here that such a task may not be extraordinary difficult, given the progress in planar silica waveguides and succesful demonstrations of thermal treatment techniques for formation of smooth curvilinear integrated optics elements. The other part of the integration is the development of appropriate waveguide coupler elements. This task can possibly be solved along three routes: a)precise tailoring of propagation constant in the waveguide to match that of the sphere; b)truncation, or "reflection" of the waveguide from vertical cleave, by analogy with the above-described fiber coupler; c)deposition of appropriate modulation grating on the waveguide (see recent report on free-space coupling to microspheres using UV-assisted surface grating [11]).

The research described in this paper was carried out, in part, by the Jet Propulsion Laboratory, California Institute of Technology, under a contract with the National Aeronautics and Space Administration.

[1] V.B.Braginsky, M.L.Gorodetsky, V.S.Ilchenko, Phys.Lett. A37, 393 (1989); H.Mabuchi, H.J.Kimble, Opt.Lett.19, 749 (1994); V.Sandoghdar, F.Treussart, J.Hare, V.Lefevre-Seguin, J.-M.Raimond, S.Haroche, Phys.Rev.A54, R1777 (1996);

[2] D.W.Vernooy, A.Furusawa, N.P.Georgiades, V.S.Ilchenko, H.J.Kimble, Phys.Rev., A57, pp.R2293-6, 1998; M.Nagai, F.Hoshino, S.Yamamoto, R.Shimano, M.Kuwata-Gonokami, Opt.Lett., 22, pp.1630-32, 1997

[3] V.V.Vasilev, V.L.Velichansky, M.L.Gorodetcky, V.S.Ilchenko, L.Hollberg, A.V.Yarovitsky, Opt.Commun. 158, 305 (1998); B.E.Little, S.T.Chu, H.A.Haus, Opt.Lett. 23, 894 (1998)

[4] M.L.Gorodetsky, A.A.Savchenkov, V.S.Ilchenko, Opt.Lett. 21, 453 (1996); D.W.Vernooy, V.S.Ilchenko, H.Mabuchi, E.W.Streed, H.J.Kimble, Opt.Lett. 23, 247 (1998)

[5] S. Todoroki, S. Sakaguchi, M. Peeters, J. Amer. Ceramic Soc., 80, pp.313-6, 1997.

[6] M.L.Gorodetsky, V.S.Ilchenko, J.Opt.Soc.Am. B16, 147 (1999)

[7] A.Serpenguezel, S.Arnold, G.Griffel, Opt.Lett. 20, 654 (1995); N.Dubreuil, J.C.Knight, D.Leventhal, V.Sandoghdar, J.Hare, V.Lefevre, Opt.Lett. 20, 813 (1995)

[8] J.C.Knight, G.Cheung, F.Jacques, T.A.Birks, Opt.Lett. 15, 1129 (1997)

[9] C.C.Lam, P.T.Leung, K.Young, J.Opt.Soc.Am. B9, 1585 (1992)

[10] X.S.Yao, L.Maleki, Opt.Lett., 22, pp.1867-9, 1997

[11] V.S.Ilchenko, D.S.Starodubov, M.L.Gorodetsky, L.Maleki, J.Feinberg, CTuC4 presented at CLEO/QELS, Baltimore, May 23-28, 1999 